\title{
Reputation-based mutual selection rule promotes cooperation in spatial threshold public goods games
}

Xiaofeng Wang

Xiaojie Chen (chenx@iiasa.ac.at)

Jia Gao

Long Wang

\section{Approved by}

Ulf Dieckmann

Director, Evolution and Ecology Program

June 2015 


\title{
Reputation-based mutual selection rule promotes cooperation in spatial threshold public goods games
}

\author{
Xiaofeng Wang ${ }^{\mathrm{a}}$, Xiaojie Chen ${ }^{\mathrm{b}}$, Jia $\mathrm{Gao}^{\mathrm{a}}$, Long Wang ${ }^{\mathrm{c}}$ \\ ${ }^{a}$ Center for Complex Systems, School of Mechano-electronic Engineering, Xidian \\ University, Xi'an Shaanxi 710071, China \\ ${ }^{b}$ Evolution and Ecology Program, International Institute for Applied Systems Analysis \\ (IIASA), Schlossplatz 1, A-2361 Laxenburg, Austria \\ ${ }^{c}$ Center for Systems and Control, State Key Laboratory for Turbulence and Complex \\ Systems, College of Engineering, Peking University, Beijing 100871, China
}

\begin{abstract}
Most previous studies involving public goods games are investigated under a simplifying assumption that participation is either compulsive or unidirectional optional in collective interactions. Nevertheless, how the mutual selection rule, a more realistic participation mode, affects the evolution of cooperation in structured populations is still unclear. Here we introduce a reputation-based mutual selection rule for constituting participant groups into spatial threshold public goods games, where the public goods game can be conducted only if the participant number is not less than the threshold parameter. Interestingly, we find that moderate tolerance range results in the best environment for cooperators' viability. Also, we show that lower member threshold is favorable for the evolution of cooperation, and correspondingly provide some typical snapshots for defectors, "active" cooperators (cooperators on which the public goods games are successfully conducted), and "inactive" cooperators. Moreover, we investigate the effects of memory factor in individuals' reputation updating on the evolution of cooperation. Our work may provide an appropriate and alternative perspective in understanding the widespread cooperative behaviors in some realistic situations.

Keywords:

Evolutionary Game theory, Cooperation, Reputation, Public Goods Game
\end{abstract}




\section{Introduction}

Cooperation behavior is crucial and ubiquitous, not only in human society but also in nature. How cooperators can survive and persist in a population of selfish individuals is an evolutionary puzzle in biology, economy and social science [1]. To address this problem, evolutionary game theory has provided a powerful framework [2]. While the prisoner's dilemma game (PDG) has become a paradigm for studying the evolution of cooperation among individuals through pairwise interactions $[1,3,4,5,6,7,8,9]$. As a natural extension, the $N$-person PDG, namely, the public goods game (PGG) for collective interactions also attracts much attention $[10,11,12,13,14,15,16,17,18,19,20]$. In a typical PGG, each player in a group of $N$ individuals is entitled to adopt either cooperation $(C)$ or defection $(D)$. A cooperator donates an investment $c(c>0)$ into the common pool, whereas a defector contributes nothing. The sum is multiplied by an enhancement factor $r(1<r<N)$ and then distributed equally among group members. Obviously, if all members have cooperated, the payoff of the group is maximized. But defection is always a better choice than cooperation for a given individual in any group. The social dilemma results as the interests of individuals do not coincide with the interests of the group, which threatens to evolve to the "Tragedy of the Commons" [21].

To escape hatch out of the evolutionary stalemate, several mechanisms have been proposed, such as costly punishment [22, 23, 24, 25, 26], voluntary participation $[27,10,11]$ and social diversity $[12,28,29]$. In ref. [22], the authors investigated the influence of altruistic punishment on the evolution of cooperation in the absence of repeated interactions by empirical experiments. They found that costly punishment is in favor of increasing the average investment of the groups. It should be noted that individuals should have some way of discriminating defectors in this work. Different from this assumption, voluntary participation was introduced in refs. $[27,10]$ by adding the third strategy 'loner' besides cooperation and defection. This simple mechanism can effectively prevent defectors from exploiting cooperators, and leads to rock-scissors-paper type cyclic dominance of the three strategies. In ref. [12], the authors have studied the PGGs on square lattice and scale-free networks, respectively. It is found that not only the diversity associated with the number and the size of PGGs, but also the diversity of individual contribution to each group can promote cooperation. All these research results provide us with novel point of view to understand the large-scale cooperation in nature 
and human society.

In previous studies on PGGs, players either are enforced to participate in all the PGGs that centered on his neighbors and himself $[23,12,28,15]$, or play PGGs optionally $[27,10,24,11]$, or are selected as group members unidirectionally by the focal individuals [30]. However, groups are not always constituted in the ways described above. Maybe a more general situation is that both the focal players and their neighbors have the privilege to choose each other as their group members [31], similar to the mutual selection rule in real life. Due to the mutual selection rule, we observe that people with similar phenotype [32, 33, 34, 35], e.g., status and characters, are more likely to interact with each other in human society. Herein, as an important partner selection criterion, reputation can also be taken as a phenotypic feature to discriminate individuals [36]. Indeed, in human society people with similar reputation tend to form partner relationship to make interactions. They not only interact with others with higher reputation, but also interact with others with lower reputation. However, individuals generally have a certain tolerance range, and do not tend to interact with the ones whose reputation is beyond the tolerance range. To our knowledge, such bidirectional selection mechanism is merely studied in the PDG under pairwise interactions [7]. Nevertheless, how such mutual selection regime affects the evolution of cooperation is still unclear in PGG under collective interactions. Furthermore, to accomplish a certain task in PGGs, perhaps the number of community members should not be less than some fixed value. In reality, if the task is too heavy to be completed for members in a group (Namely, there are not enough members to bear the task.), these players will naturally give up the chance of playing the game to avoid the certain loss. The examples of such cases are hunting behavior of predators, building construction and public transportation. Thus it is reasonable that there exists some member threshold in PGGs [37]. In view of the above situations, presently we consider individual tolerance range and reputation to construct a mutual selection mechanism into spatial threshold PGGs. Interestingly, we find that moderate tolerance range results in the best environment for cooperators' viability. Besides, we show that smaller member threshold is favorable for the evolution of cooperation.

The rest of the paper is organized as follows. First, we describe the spatial threshold PGG and the protocol for mutual selection mechanism. Then we present the results, whereas lastly we give some discussion and draw our conclusion. 


\section{Model}

Now let us briefly introduce our model. At the start of evolution, each player, who occupies one site of the square lattice with periodic boundary conditions, adopts one of the two pure strategies: cooperation $(C)$ and defection $(D)$, with equal probability. In each generation, each player $i$ tends to pick out his neighbor $j$ in its Moore neighborhood if both of them are in the tolerance range of each other's reputation, that is, $\left|R_{i}-R_{j}\right| \leqslant h$, where $R_{i}\left(R_{j}\right)$ is the reputation score of player $i(j)$, and $h$ is the tolerance range of players $i$ and $j$. If the number $\left(m_{i}\right)$ of group members participating in PGG that centered on player $i$ is not less than the member threshold $T$, namely, $m_{i} \geqslant T$, the PGG can be conducted successfully. Otherwise, the PGG breaks down. For simplicity, we assume that each player has the same tolerance range and each group has identical member threshold in this paper.

According to the rule described above, player $i$ engages in threshold PGGs , and the payoff $P_{i j}$ associated with group centered on its neighbor $j$ and itself is given by

$$
P_{i j}=\left\{\begin{array}{ll}
0, & \text { if } m_{j}<T \\
\frac{r m_{c j}}{m_{j}}-S_{i}, & \text { if } m_{j} \geq T
\end{array},\right.
$$

where $r$ indicates the enhancement factor, $S_{i}$ denotes the strategy of individual $i\left(S_{i}=1\right.$ for $C ; S_{i}=0$ for $\left.D\right)$, and $m_{c j}$ is the number of cooperators among participants in group $j$. Here the cost is normalized to unity. The total payoff individual $i$ thereby acquires is the sum of the payoffs received in all the groups: $P_{i}=\sum_{j} P_{i j}$.

After playing the game, each player updates its reputation score. An individual's reputation score is assessed as a weighted average of its previous score of reputation and its immediately preceding experience with its interaction neighbors. Thus the reputation score of player $i$ at time step $t$ is

$$
R_{i}(t)=\alpha \frac{S_{i}(t) N_{i}(t)}{k_{i}+1}+(1-\alpha) R_{i}(t-1),
$$

where $0<\alpha<1$ is a weighting factor, $\left(k_{i}+1\right)$ denotes the maximal possible number of PGGs player $i$ participates $\left(k_{i}+1=9\right.$ in present model), and $N_{i}(t)$ is the actual number of PGGs player $i$ participates at time $t$. $S_{i}(t) N_{i}(t)$ represents the frequency of player $i$ 's altruistic behaviors, while $\frac{S_{i}(t) N_{i}(t)}{k_{i}+1}$ normalizes the number of times of player $i$ 's altruistic behaviors. $R_{i}(t-1)$ denotes the reputation score of player $i$ at time $t-1$. 
Afterwards, players will adjust their strategies according to the following updating rule: player $i$ randomly selects one of his neighbors, $j$, and $i$ adopts $j$ 's strategy with a probability [38]:

$$
T\left(S_{j} \rightarrow S_{i}\right)=\frac{1}{1+\exp \left[\left(P_{i}-P_{j}\right) / \kappa\right]},
$$

where $\kappa$ characterizes the amplitude of noise. For $\kappa=0, i$ will adopt $j$ 's strategy deterministically when $P_{i}<P_{j}$. For $\kappa>0$, there exists a certain possibility that strategies performing worse will be adopted. In most cases, selfish and rational individuals prefer the strategies of more successful neighbors. In present work, we simply fix $\kappa=0.1$.

\section{Results}

Simulations are carried out for a population of size $N=30 \times 30$ on a square lattice with periodic boundary conditions. It should be noted that the main results remain unchanged for different population sizes. Initially, the two strategies of $C$ and $D$ are randomly distributed among the players of the population with equal probability. For convenience of simulation tractability, each player's reputation is uniformly distributed within the interval $[0,1]$ at the start of evolution. When the system reaches equilibrium, the fraction of cooperators $\rho_{c}$ in the whole population is calculated as a key quantity for measuring the evolution of cooperation. In all the simulations, the fraction of cooperators $\rho_{c}$ is obtained by averaging over the last $2 \times 10^{3}$ Monte Carlo time steps of the total $2 \times 10^{4}$. Synchronous update rule based on pairwise comparison for a fixed noise level (i.e., $\kappa=0.1$ ) is adopted for strategy evolution. In what follows, we investigate how the tolerance range $h$ affects the evolution of cooperation. Moreover, the effects of member threshold $T$ and memory factor $\alpha$ are also studied.

Figure 1 shows how cooperation evolves depending on the enhancement factor $r$ (a) and reputation tolerance $h$ (b), respectively. In fig. 1 (a), it is clearly shown that the cooperation level $\rho_{c}$ monotonously increases with the enhancement factor $r$ for each value of $h$ [16]. Interestingly, compared with other cases, the one with $h=0.4$ leads to the optimal cooperation level [see fig. 1 (a)]. It indicates that there may exist some intermediate values of $h$, resulting in the most idealistic scenario for cooperators. To further investigate the influence of $h$ upon the cooperation level, we study the cooperator density $\rho_{c}$ versus tolerance range $h$ for various $r$ [see fig. 1 

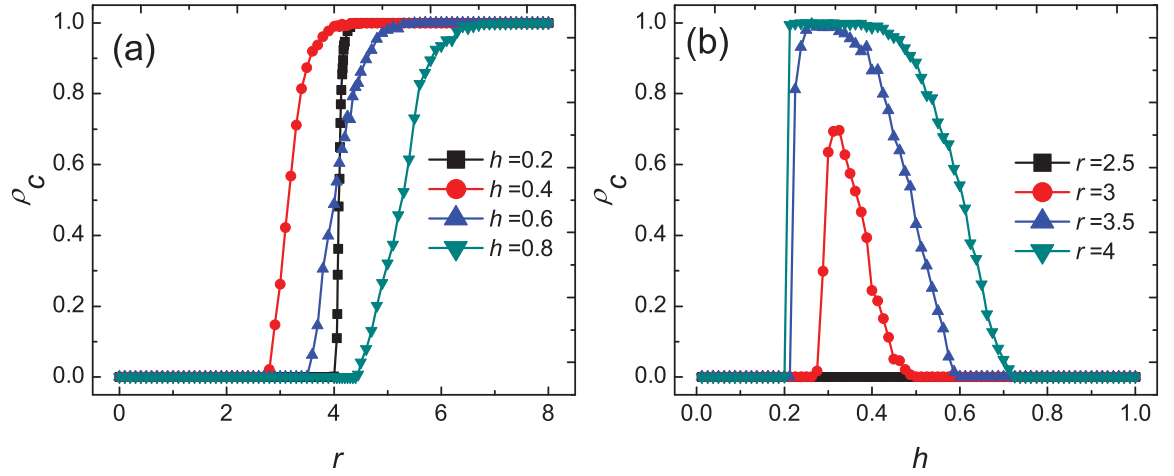

Figure 1: (Color online) Evolution of cooperation. (a) Fraction of cooperators $\rho_{C}$ as a function of $r$ for different values of $h$. (b) Fraction of cooperators $\rho_{C}$ as a function of $h$ for different values of $r$. Here $\alpha$ is set to 0.5 and $T$ is set to 6 . Each data point results from $9 \times 10^{2}$ independent initial realizations.

(b)]. It can be observed that there exist moderate values of $h$, leading to a plateau of high cooperation level for large values of $r$. With decreasing $r$, the length of plateau diminishes. While the value of $r$ decreases to 3 , the plateau vanishes. However, it can still be observed that a modest value of $h$ induces an optimal cooperation level. Finally when $r=2.5$, the cooperation level approaches to zero irrespective of $h$ [see fig. 1 (b)].

To understand the evolution of cooperation under such reputation-based mutual selection mechanism more intuitively, we report the variation of fraction of cooperators $\rho_{C}$, the average number of participators with strategy $j$ in PGGs centered on participators with strategy $i N_{i j}$ (i.e., $N_{C C}, N_{C D}, N_{D C}$ and $N_{D D}$ ) and the average frequency of participators with strategy $i$ participating in PGGs $M_{i}$ (i.e., $M_{C}$ and $M_{D}$ ) with respect to period in fig. 2. For small $h$ (e.g., $h=0.2$ ), the interactions between cooperators via participating in PGGs are limited due to the different reputations of cooperators [see fig. 2 (c)]. This in turn leads to the decline of cooperators' reputations. As a result, cooperators can not effectively filter defectors via the mutual selection mechanism, as the reputations of cooperators and defectors are not heterogeneous enough compared with the tolerance range $h$ [see fig. 2 (b)]. Whereas for defectors, owing to low reputation, they are more likely to recruit enough members to participate in their PGGs [see fig. 2 (c)], though most of their group members are defectors [see fig. 2 (b)]. As a result, cooperators can not help each other, and are wiped out by defectors eventually [see fig. 2 (a)]. 

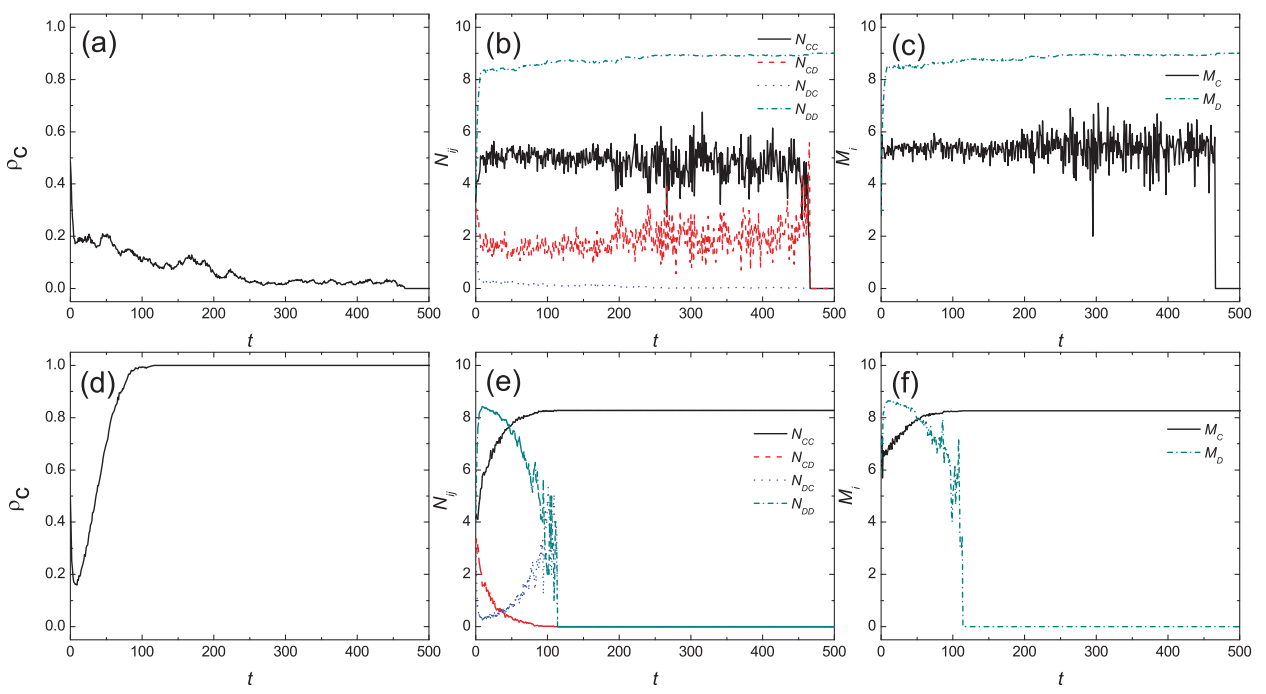

Figure 2: (Color online) Time evolution of the fraction of cooperators $\rho_{C}$, the average number of participators with strategy $j$ in PGGs centered on participators with strategy $i$ $N_{i j}\left(N_{C C}, N_{C D}, N_{D C}\right.$ and $\left.N_{D D}\right)$ and the average frequency of participators with strategy $i$ participating in PGGs $M_{i}\left(M_{C}\right.$ and $\left.M_{D}\right): h=0.2[(\mathrm{a}),(\mathrm{b})$ and $(\mathrm{c})]$ and $h=0.4[(\mathrm{~d}),(\mathrm{e})$ and (f)]. Other parameter values are $r=4, \alpha=0.5$, and $T=6$, respectively. The data shown here are obtained in one realization. 


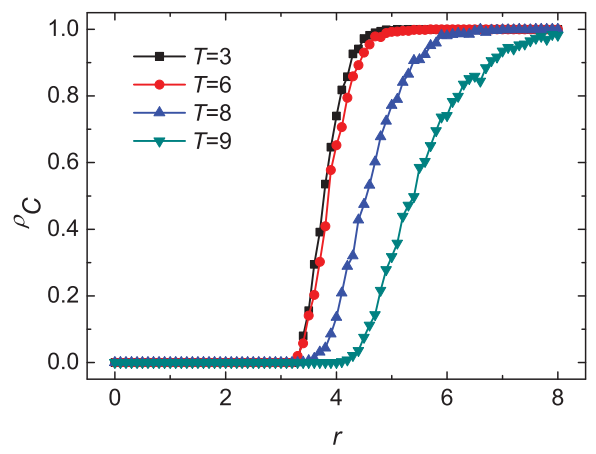

Figure 3: (Color online) Fraction of cooperators $\rho_{C}$ as a function of enhancement factor $r$ for member threshold $T=3,6,8$ and 9 , respectively. Other parameters are $h=0.5$ and $\alpha=0.5$. Each data point results from $9 \times 10^{2}$ independent initial realizations.

While for intermediate $h$ (e.g., $h=0.4$ ), on one hand, cooperators are able to protect themselves by preventing defectors with low reputation from engaging in their groups [see fig. 2 (e)]. On the other hand, compared with small $h$, cooperators can engage in more PGGs to play with conspecifics [see fig. 2 (f)]. Furthermore, defectors are restricted to play PGGs with their defective neighbors [see fig. 2 (e) and 2 (f)]. The overall effects induce a positive feedback mechanism, which allows for the reciprocal interactions between cooperators becoming larger and stronger. Then compact cooperator clusters form and expand to the whole population [see fig. 2 (d)]. Finally for large $h$, each individual has little restriction to interact with each other. In the case that $r$ is not large enough, defectors perform better than cooperators, and thus dominate the population finally [see fig. 1 (b)].

In what follows, we will focus on the influence of member threshold upon the evolution of cooperation. As shown in fig. 3, compared with the case of large values of member threshold $T$, smaller $T$ clearly promotes the evolution of cooperation. To give an intuitive explanation to cooperators' different evolutionary fates for various values of $T$, we report the evolution of the spatial distribution of players at different periods for $T=5$ and $T=9$, respectively in fig. 4. For convenience of illustration, we distinguish a cooperator based on whether or not the PGG centered on herself is conducted successfully. In accordance with this criterion, cooperators can be classified as being either "active" or "inactive" [39]. Due to the random distribution of players and reputation at the beginning of evolution [see fig. 4 (a) and 4 (i)], on one hand, cooperators scatter dispersedly in the network, and defectors permeate 
the whole population. On the other hand, cooperators can not discriminate defectors through their randomly distributed reputations. As a result, cooperators can not escape the exploitation of defectors in the most early stages of evolutionary process, irrespective of the values of $T$ (see fig. 4).

Intriguingly, if the member threshold is large (e.g., $T=9$ ), most cooperators are "inactive" at the beginning. Compared with lower $T$, higher $T$ is in favor of cooperators for its restricted interaction with defectors at the start of evolution (see fig. 4). Nonetheless, the "inactive" behaviors of cooperators result in their decline of reputation scores as those of defectors. Hence most cooperators are activated to play PGGs with defectors at the next time step leading to the reduction of cooperator density. Then the majority of cooperators become "inactive" again [see fig. $4(\mathrm{i})-4(\mathrm{k})$ ]. Interestingly, cooperators switch between "active" and "inactive" state in circles. This phenomenon is similar to the "ping-pong effect" as reported in refs. [40]. However, cooperators can not escape the fate of extinction eventually [see fig. 4 (p)]. While for small $T$ (e.g., $T=5$ ), the majority of cooperators are "active" in the beginning [see fig. 4 (a)]. Most "active" cooperators are vulnerable in the presence of defectors at the start of evolution [see fig. 4 (a)-4 (d)]. But along with the evolutionary process moving forward, the reputations of the individuals, who frequently exploit the public goods, gradually become inferior to that of the few contributors left. Thus these individuals with low reputations will be banished by cooperators through the mechanism of mutual selection. Namely, the aggregation of cooperators works. Herein we argue that once the positive assortments of cooperators works, smaller member threshold $T$ is better for cooperators' viability [see fig. 4 (e)-4 (h)]. On one hand, smaller member threshold $T$ induces dynamic group size, which determines a richer spectrum of individual payoffs. On the other hand, compared with the case of large $T$, the social dilemma of groups with smaller sizes existing in the case of smaller $T$ is more relaxed. As a result, cooperators have higher probability to survive with small $T$ than that they have with large $T$ (see fig. 3 ). Intriguingly, our findings agree with the results reported in ref. [12]. The authors reported that in the case each player invests a fixed cost per game, both the diversity in the size of each player's PGG and the number of PGGs in which each player participates induced by the heterogeneity of the underlying networks facilitate the evolution of cooperation. Our findings confirm their research results. While in our work, the diversity for the evolution of cooperation is induced by the mutual selection mechanism and member threshold, rather than the network structure. For further study, we think it 
is interesting to study the properties of patterns about these "active" and "inactive" cooperators.

Finally, we would like to discuss the effect of memory (i.e., weighting factor $\alpha$ ) upon the evolutionary fate of cooperators. Figure 5 shows the cooperation level as a function of tolerance range $h$ for different values of $\alpha$. Clearly, in each case, there still exists intermediate values of $h$, which result in the best environment for viability of cooperators. Moreover, for small $h$, smaller weighting factor $\alpha$ induces better chance for the survival of cooperators, while larger and moderate $\alpha$ is more favorable for the evolution of cooperation in the case of large and intermediate values of $h$, respectively. Actually, it is not difficult to explain those phenomena. While the values of $\alpha$ are small, individuals' reputation mainly depends on the historical performance, which slows down the separation process of the reputation scores of cooperators and defectors, but indicates the exact information regarding the cooperativity of their neighbors in all past games. In this situation, for small $h$, on one hand, cooperators can protect themselves by restricting interaction with defectors. On the other hand, due to the exact information induced by small $\alpha$, cooperators can distinguish each other more accurately. Hence while $h$ is small, smaller $\alpha$ is better for cooperators to help each other (see fig. 5). However, when $\alpha$ is large, player's reputation mainly relies on the current performance, which introduces a rapid feedback mechanism between the reputation score and the present performance. But due to the different number of groups in which each cooperator participates, the reputation score of cooperators dissimilates. As a result, the interaction between cooperators will be inhibited for small $h$. While for large $h$, cooperators interact with each other with little restriction (see fig. 5). Thus in the case of large $h$, larger $\alpha$ is more beneficial for cooperators to evolve. While $h$ is intermediate, as one can expect, moderate values of $\alpha$ induces the best environment for the evolution of cooperation (see fig. 5).

\section{Discussion and conclusion}

Recently, Chen and Wang studied the PDG by considering the mutual selection mechanism in the Barabási-Albert scale-free networks [7]. They found that moderate tolerance range can result in the best promotion of cooperation. It should be noted that our model is different from theirs. In our model, the mutual selection mechanism is studied on a square lattice in the context of collective interaction. Hence, we not only can explore 

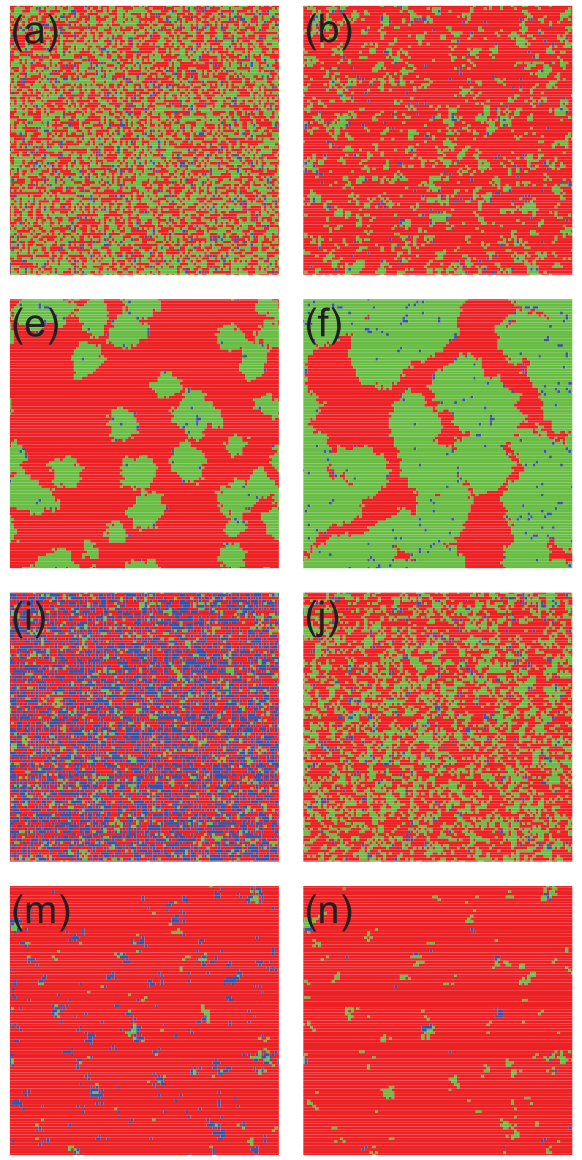
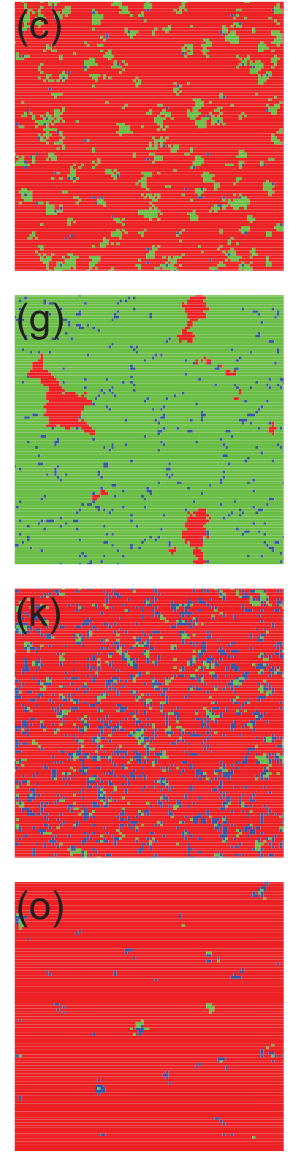

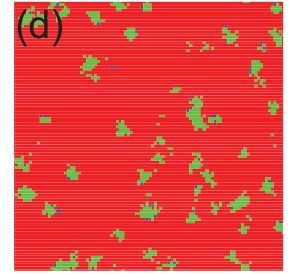

Figure 4: (Color online) Time evolution of distributions of players on a square lattice for $T=5$ [(a) $t=0$, (b) $t=2$, (c) $t=4$, (d) $t=8$, (e) $t=50$, (f) $t=100$, (g) $t=150$, and (h) $t=200]$ and $T=9$ [(i) $t=0$, (j) $t=1$, (k) $t=2$, (l) $t=3$, (m) $t=4$, (n) $t=5$, (o) $t=6$, and (p) $t=7$ ] at $r=4$. Blue and green represent inactive and active cooperators, respectively, while red denotes defectors. These snapshots show a $100 \times 100$ square lattice. 


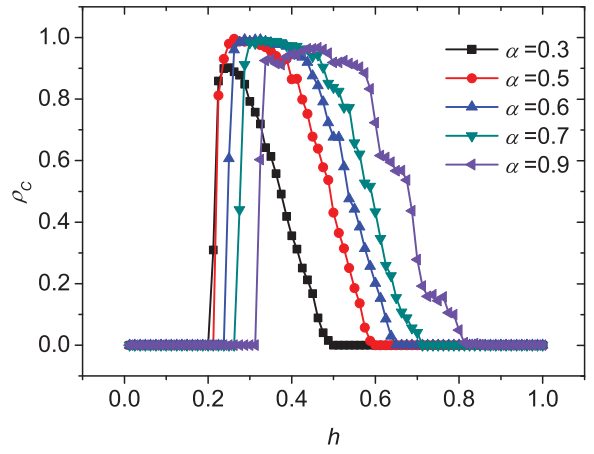

Figure 5: (Color online) Fraction of cooperators $\rho_{C}$ as a function of $h$ for different values of $\alpha$. Here $r$ is set to 3.5 and $T$ is set to 6 . The results are obtained in $9 \times 10^{2}$ independent initial realizations.

the effect of reputation tolerance more directly in a homogeneous networked environment, but also can study how the mutual selection mechanism works in the context of group interaction. Moreover, in this work we consider a member threshold parameter, that is, only if the number of participants approaches the member threshold the public good game is conducted. It is worth pointing out that this threshold parameter is different from the one in refs. [14, 16, 41, 39], where the public good is produced only if the number of cooperators is not less than the threshold. Correspondingly, the payoff function in our model is different from the step gain all-or-nothing function in other related threshold PGG models. Under this mutual selection rule in threshold PGGs, the participant group for collective interaction may shrink and diversity of interaction groups emerges even in a homogeneous graph, which are demonstrated to support the evolution of cooperation [12]. In a sense, our study further confirms and compensates the positive effects of moderate tolerance on the evolution of cooperation in a more realistic and complicated environment.

In summary, we have studied the spatial threshold PGGs by considering mutual selection mechanism based on individual reputation and tolerance. We have shown that such mutual selection results in the optimal cooperation level at moderate values of tolerance range. In addition, we have investigated the effect of member threshold in PGGs. Although cooperative behavior initially dose seem to fare better under the regime of large member threshold, in the long run the evolution of cooperation is impeded due to the homogeneity of defectors' and cooperators' reputation. While smaller member threshold 
induces nasty environment for cooperators at the very infancy of the games, but it is, after all, good for cooperator's viability in a long term. Interestingly, via the classification of cooperators as being either "active" or "inactive", an intriguing "ping-pong effect" emerges in the case of high member threshold. Finally, we studied the influence of memory on the evolution of cooperation. We found that there exists an optimal tolerance range leading to the highest cooperation density for each value of weighting factor. Furthermore, it is found that small values of weighting factor are more favorable for cooperators' viability when tolerance rang is small; intermediate values of weighting factor are better for the evolution of cooperation when tolerance range is moderate; and large values of weighting factor are beneficial for the survival of cooperators when tolerance range is large.

\section{Acknowledgement}

The authors are supported by National 973 Program (2012CB821203) and NSFC (61020106005, 11161011, 61203374, and 10972002).

\section{References}

[1] Axelrod R. The Evolution of Cooperation. New York: Basic Books; 1984

[2] Maynard Smith J. Evolution and the Theory of Games. Cambridge, UK: Cambridge Univ. Press; 1982.

[3] Santos FC, Rodrigues JF, Pacheco JM. Epidemic spreading and cooperation dynamics on homogeneous small-world networks. Phys Rev E 2005;72:056128-5.

[4] Chen XJ, Fu F, Wang L. Influence of different initial distributions on robust cooperation in scale-free networks: A comparative study. Phys Lett A 2008;372:1161-7.

[5] Fu F, Hauert C, Nowak MA, Wang L. Reputation-based partner choice promotes cooperation in social networks. Phys Rev E 2008;78:026117-8.

[6] Fu F, Wu T, Wang L. Partner switching stabilizes cooperation in coevolutionary prisoner's dilemma. Phys Rev E 2009;79:036101-7. 
[7] Chen XJ, Wang L. Cooperation enhanced by moderate tolerance ranges in myopically selective interactions. Phys Rev E 2009;80:046109-6.

[8] Wang XF, Perc M, Liu YK, Chen XJ, Wang L. Beyond pairwise strategy updating in the prisoner's dilemma game. Sci Rep 2012;2:740-8.

[9] Liu YK, Zhang L, Chen XJ, Ren L, Wang L. Effect of community structure on coevolutionary dynamics with dynamical linking. Physica A 2013;392:3640-7.

[10] Hauert C, De Monte S, Hofbauer J, Sigmund K. Volunteering as Red Queen mechanism for cooperation in public goods game. Science 2002;296:1129-32.

[11] Szabó G, Hauert C. Phase transitions and volunteering in spatial public goods games. Phys Rev Lett 2002;89:118101-4.

[12] Santos FC, Santos MD, Pacheco JM. Social diversity promotes the emergence of cooperation in public goods games. Nature 2008;454:2136.

[13] Wu T, Fu F, Wang L. Individual's expulsion to nasty environment promotes cooperation in public goods games. EPL 2009;88:30011-6.

[14] Wang J, Fu F, Wu T, Wang L. Emergence of social cooperation in threshold public good games with collective risk. Phys Rev E 2009;80:016101-11.

[15] Helbing D, Szolnoki A, Perc M, Szabó G. Defector-accelerated cooperativeness and punishment in public goods games with mutations. Phys Rev E 2010;81:057104-4.

[16] Boza G, Számadó S. Beneficial laggards: multilevel selection, cooperative polymorphism and division of labour in threshold public good games. BMC Evol Biol 2010;10:336-12.

[17] Perc M, Gómez-Gardeñes J, Szolnoki A, Floría LM, Moreno Y. Evolutionary dynamics of group interactions on structured populations: a review. J R Soc Interface 2013;10:80-17. 
[18] Liu Y, Chen X, Zhang L, Tao F, Wang L. Social selection of game organizers promotes cooperation in spatial public goods games. EPL 2013;102:50006-6.

[19] Chen X, Szolnoki A, Perc M, Wang L. Impact of generalized benefit functions on the evolution of cooperation in spatial public goods games with continuous strategies. Phys Rev E 2012;85:066133-7.

[20] Wu T, Fu F, Zhang Y, Wang L. Expectation-driven migration promotes cooperation by group interactions. Phys Rev E 2012;85:066104-9.

[21] Hardin G. The tragedy of the commons. Science 1968;162:1243-8.

[22] Fehr E, Gächter S. Altruistic punishment in humans. Nature 2002;415:137-40.

[23] Brandt H, Hauert C, Sigmund K. Punishment and reputation in spatial public goods games. Proc R Soc Lond B 2003;270:1099-104.

[24] Hauert C, Traulsen A, Brandt H, Nowak MA, Sigmund K. Via freedom to coercion: The emergence of costly punishment. Science 2007;316:1905-7.

[25] Helbing D, Szolnoki A, Perc M, Szabó G. Punish, but not too hard: how costly punishment spreads in the spatial public goods game. New J Phys 2010;12:083005-14.

[26] Perc M. Sustainable institutionalized punishment requires elimination of second-order free-riders. Sci Rep 2012;2:344-6.

[27] Hauert C, De Monte S, Hofbauer J, Sigmund K. Replicator dynamics in optional public goods games. J Theor Biol 2002;218:187-94.

[28] Perc M, Szolnoki A. Social diversity and promotion of cooperation in the spatial prisoner's dilemma game. Phys Rev E 2008;77:011904-5.

[29] Gao J, Li Z, Wu T, Wang L. Diversity of contribution promotes cooperation in public goods games. Physica A 2010;389:3166-71.

[30] Wu T, Fu F, Wang L. Partner selections in public goods games with constant group size. Phys Rev E 2009;80:026121-8. 
[31] Coricelli G, Fehr D, Fellner G. Partner selection in public goods experiments. J Conflict Resol 2004;48:356-78.

[32] Jansen VAA, Baalen M. Altruism through beard chromodynamics. Nature 2006;440:663-6.

[33] Traulsen A, Nowak MA. Chromodynamics of cooperation in finite populations. PLoS ONE 2007;2:e270-6.

[34] Antal T, Ohtsuki H, Wakeley J, Taylor PD, Nowak MA. Evolutio of cooperation by phenotypic similarity. Proc Natl Acad Sci USA 2009;106:8597-600.

[35] Tarnita CE, Antal T, Ohtsuki H, Nowak MA. Evolutionary dynamics in set structured populations. Proc Natl Acad Sci USA 2009;106:8601-4.

[36] Nowak MA, Sigmund K. Evolution of indirect reciprocity by image scoring. Nature 1998;393:573-7.

[37] Haag M, Lagunoff R. On the size and structure of group cooperation. J Econ Theor 2007;135:68-89.

[38] Szabó G, Tőke C. Evolutionary prisoner's dilemma game on a square lattice. Phys Rev E 1998;58:69-73.

[39] Szolnoki A, Perc M. Impact of critical mass on the evolution of cooperation in spatial public goods games. Phys Rev E 2010;81:057101-4.

[40] Chen XJ, Wang L. Promotion of cooperation induced by appropriate payoff aspirations in a small-world networked game. Phys Rev E 2008; 77:017103-4.

[41] Souza MO, Pacheco JM, Santos FC. Evolution of cooperation under n-person snowdrift games. J Theor Biol 2009;260:581-8. 